\title{
Advantages of using reduced-volume intensity modulated radiation therapy for the treatment of nasopharyngeal carcinoma: a retrospective paired study
}

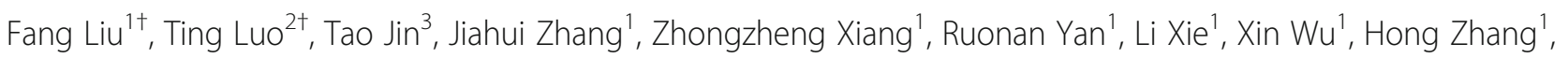
Feng Wang ${ }^{1}$, Ping Li' ${ }^{1}$ and Lei Liu ${ }^{1 *}$

\begin{abstract}
Background: The definition of clinical target volume (CTV) in intensity modulated radiation therapy (IMRT) for nasopharyngeal carcinoma (NPC) has not been addressed. We performed this study to assess the feasibility and efficacy of using IMRT with reduced-volume CTV for the treatment of nasopharyngeal carcinoma.

Methods: We retrospectively reviewed 293 non-metastatic NPC patients treated with IMRT from 2002 to 2013. A total of 180 matched cases finally included with 90 received conventional-volume IMRT (CV-IMRT) and 90 received reducedvolume IMRT (RV-IMRT). Kaplan-Meier method and log-rank tests were used to compare NPC-specific survival. Multivariate analyses using the Cox proportional hazards model were conducted to detect independent predictors.

Results: With a median follow-up of 70 months, the 3-year overall survival, progression-free survival, distant metastasisfree survival, local recurrence-free survival, regional recurrence-free survival, locoregional recurrence-free survival rates were 88.9, 84.4, 92.2, 91.1, 98.9, 91.1\% for the CV-IMRT arm and 92.2, 85.6, 90.0, 93.3, 98.9, 93.3\% for the RV-IMRT arm, respectively. None significant survival difference was observed. Additionally, RV-IMRT was associated with reduced risk of late xerostomia $(P=0.039)$ and hearing loss $(P=0.008)$, compared versus CV-IMRT.
\end{abstract}

Conclusions: The use of RV-IMRT for the treatment of NPC led to comparable survival condition and truly reduced toxicity reactions compared versus CV-IMRT.

Keywords: Nasopharyngeal carcinoma, Radiotherapy, Intensity modulated radiation therapy, Clinical target volume

\section{Background}

Nasopharyngeal carcinoma (NPC) is a malignancy that shows high prevalence in Southeast Asia and Southern China $[1,2]$. Radiotherapy has been regarded as the most effective and only curative treatment modality for NPC [3]. The locoregional control of NPC chiefly depends on high-dose radiotherapy, whereas the adjacent critical, dose-limiting normal structures are inevitably exposed to rays. Intensity modulated radiation therapy (IMRT), a way of breaking through in the treatment of

\footnotetext{
*Correspondence: liuleihx@gmail.com

${ }^{\dagger}$ Fang Liu and Ting Luo contributed equally to this work.

'Department of Radiation Oncology, Cancer Center, State Key Laboratory of Biotherapy, West China Hospital, Sichuan University, Chengdu 610041,

Sichuan, China

Full list of author information is available at the end of the article
}

NPC, offering significant advantages in target dose uniformity and the sparing of adjacent organs at risk, causing a more satisfactory disease control and a lower rate of toxicity than traditional radiotherapy [4-6]. A number of studies [7-10] have constantly reported an over $85 \%$ local control of NPC treated with IMRT. Although IMRT has been widely used, the selection and definition of clinical target volume (CTV) in IMRT for NPC has not yet reached a consensus. The Radiation Therapy Oncology Group (RTOG) trials involving IMRT of NPC put forward the delineation of CTV [11, 12], which were largely based on our previous experience in twodimensional radiotherapy (2D-RT). According to the previous research results, locoregional recurrence has become a main failure mode and the majority occurs 
within the treatment field. Nevertheless, isolated marginal recurrence was hardly found even in advanced $\mathrm{T}$ stage cases [13-15], which signified that the wide field used in 2D-RT might be redundant. In addition, increased highdose treatment volumes almost surely increase radiation toxicity reactions, as well as the incidence of second primary tumor (SPT). The intentional coverage of all adjacent structures in CTV may be unnecessary and disputable, especially in IMRT era. Based on these, Fujian Provincial Tumor Hospital Affiliated to Fujian Medical University formulated a reduced-volume IMRT (RVIMRT) delineation [16]. To our knowledge, there has not been direct comparison between the two protocols in the treatment of NPC.

We performed this study to investigate the feasibility and efficacy of using IMRT with reduced-volume CTV for the treatment of NPC, with the hope to provide certain reference meanings for further studies.

\section{Methods \\ Patients}

Between December 2002 and August 2013, 293 histologically diagnosed, non-metastatic and treatment-naive nasopharyngeal carcinoma patients were treated with IMRT at our center. Pretreatment staging evaluation consisted of a complete physical examination, liver and renal biochemical analysis, complete blood cell count, flexible fiberoptic endoscopic examination, computed tomography (CT) scanning/magnetic resonance imaging (MRI) of the head and neck, bone scan, chest X-ray, ultrasonography of the abdomen, and dental evaluation. Positron emission tomography scans and CT scans of the chest/abdomen were performed when necessary. Tumors were staged according to the American Joint Committee on Cancer (AJCC) 2010 cancer staging classification.

\section{Radiotherapy}

Between December 2002 and December 2009, the first 122 NPC patients were treated with IMRT and the target volumes were delineated by taking RTOG protocol as the reference [11], which was regarded as the conventional-volume IMRT (CV-IMRT) group. As determined by clinical, endoscopic examinations and imaging, the gross tumor volume (GTV) included the primary nasopharyngeal tumor (GTV-P) and the involved lymph nodes (GTV-N). The clinical target volume was defined as the subclinical regions at risk for involvement. The CTV-1 was defined as GTV plus areas at risk including the entire nasopharynx, skull base, parapharyngeal space, retropharyngeal space, pterygopalatine fossae, clivus, the inferior part of the sphenoid sinus, the posterior third of the nasal cavity and maxillary sinuses, and the upper deep jugular nodes. The
CTV-2 was contoured as middle and lower jugular nodes. The planning target volumes (PTVs) were expanded by $3 \mathrm{~mm}$ from the above volumes in case of setup uncertainties and kinematic errors. Generally, a total dose of $70 \mathrm{~Gy}$ in 33 fractions at $2.12 \mathrm{~Gy} /$ fraction to the PTVs of GTV-P and GTV-N, 60 Gy in 33 fractions at $1.82 \mathrm{~Gy} /$ fraction to the PTV of CTV-1, $56 \mathrm{~Gy}$ in 33 fractions at $1.70 \mathrm{~Gy} /$ fraction to the PTV of CTV-2 were prescribed.

The reduced-volume IMRT (RV-IMRT) group enrolled the next 171 NPC patients during January 2010 to August 2013. The GTV was defined as that described for the CV-IMRT arm. The CTV-1 was contoured as GTV plus $5-10 \mathrm{~mm}$ margin, as well as the nasopharyngeal mucosa plus $5 \mathrm{~mm}$ submucosal region. The CTV-2 covered areas including the nasopharyngeal cavity (the posterior part of nasal cavity), parapharyngeal space, maxillary sinus (the anterior $5 \mathrm{~mm}$ part of the posterior nasal aperture and maxillary mucosa), posterior ethmoid sinus, the inferior part of spheniod sinus and cavernous sinus, skull base, the anterior third part of clivus and cervical vertebra, pterygopalatine fossa, and retropharyngeal lymph nodes (from skull base to cranial edge of the $\mathrm{C} 2$ vertebra). The CTV-N included levels II to V nodal regions (upper deep jugular nodes were not covered unless involved). The PTVs were expanded by $3 \mathrm{~mm}$ from the above volumes. A total dose of $70 \mathrm{~Gy}$ in 33 fractions at $2.12 \mathrm{~Gy} /$ fraction to the PTVs of GTV-P and GTV-N, $60 \mathrm{~Gy}$ in 33 fractions at $1.82 \mathrm{~Gy} /$ fraction to the PTV of CTV-1, 56 Gy in 33 fractions at $1.70 \mathrm{~Gy} /$ fraction to the PTVs of CTV-2 and CTV-N were prescribed.

No matter what kind of radiation protocol was used, critical normal structures including the spinal cord, brainstem, temporal lobes, hypophysis, optic nerves, chiasm, eyeballs, lens, parotid glands, temporomandibular joints and mandible were set as organs at risk (OARs). The dose received by each OAR was limited according to the RTOG protocol. At our center, computerized optimization was utilized with fusion of MRI with planning CT images to accurately delineate the target volumes. Treatment plans were generated using the Elekta PrecisePLAN (Release 2.10). Patients received IMRT with 6-MV X-ray beams modulated using Elekta Precise and Elekta Synergy VMAT (Elekta, Stockholm, Sweden). In general, treatment was delivered one fraction daily, 5 days per week.

\section{Chemotherapy}

Patients from both groups presented with stage II-IVb were treated with IMRT combined with cisplatin-based concurrent chemotherapy (cisplatin $80 \mathrm{mg} / \mathrm{m}^{2}$ divided into 3 parts on days $1-3$, every 3 weeks). Neoadjuvant or adjuvant chemotherapy was given at discretion of the attending physician, chemotherapy protocols including 
PF (cisplatin $80 \mathrm{mg} / \mathrm{m}^{2}$ divided into 3 parts on days $1-3$, and fluorouracil $750 \mathrm{mg} / \mathrm{m}^{2}$ per day on days $1-5$, every 3 weeks) and TPF (paclitaxel $135 \mathrm{mg} / \mathrm{m}^{2}$ day 1 , cisplatin $80 \mathrm{mg} / \mathrm{m}^{2}$ divided into 3 parts on days $1-3$, and fluorouracil $750 \mathrm{mg} / \mathrm{m}^{2}$ per day on days $1-5$, every 3 weeks).

\section{Follow-up}

All patients were required to be followed up after the completion of treatment: every 3 months in the first 2 years, every 6 months over the following 3 years, and then annually thereafter. Each follow-up consisted of physical examination, flexible fiberoptic endoscopy, ultrasound of abdomen, chest X-ray, and basic serum chemistry. Either CT or MRI scans of the head and neck were performed after the completion of radiotherapy and then when clinically needed to evaluate the treatment response. Late toxicities were defined as symptoms occurred beyond 3 months after the completion of treatment and were assessed at each follow-up according to the Common Terminology Criteria for Adverse Events (CTCAE v4.0) [17].

\section{Statistical analysis}

To minish the interference of heterogeneity, patients were paired using propensity score matching (PSM) method [18]. Propensity scores for each patient were computed based on the following covariates: sex, age, Tstage, N-stage, clinical stage, use of chemotherapy (concurrent chemotherapy, neoadjuvant or adjuvant chemotherapy). Patients were then matched at the ratio of 1:1 to create similar case and control arms with balanced characteristics. The balance between the two arms was examined by chi-square test or rank sum test (ranked data).

In this study, overall survival (OS), progression-free survival (PFS), distant metastasis-free survival (DMFS), local recurrence-free survival (LRFS), regional recurrencefree survival (RRFS) and locoregional recurrence-free survival (LRRFS) were analyzed as the endpoints. The duration of time to recurrence and distant metastasis was counted from the completion of radiotherapy until treatment failure. The duration of OS was measured from diagnosis until death or until the last follow-up for patients still alive. For locoregional recurrence cases, in-field failure was determined as $95 \%$ or more of the recurrence volume within the $95 \%$ isodose. Marginal failure was defined as 20 to $95 \%$ of the recurrence volume within the $95 \%$ isodose. Out-field failure was defined as less than $20 \%$ of the recurrence volume within the $95 \%$ isodose. OS, PFS, DMFS, LRFS, RRFS and LRRFS were estimated using the Kaplan-Meier method [19] and pairwise comparisons between groups were calculated using log-rank tests. To detect independent predictors, multivariate analyses were performed using the Cox proportional hazards model.
Rank sum test was adopted to compare the adverse events.

All statistical analyses were conducted using IBM SPSS Statistics version 23.0. All tests were two sided, and a $P$ value of less than 0.05 was considered statistically significant.

\section{Results}

\section{Patient clinical characteristics}

Of the 293 untreated non-metastatic NPC patients, 180 paired cases finally included with 90 received CV-IMRT and 90 received RV-IMRT. There were 12 (6.7\%), 32 (17.8\%), 83 (46.1\%), and $53(29.4 \%)$ patients presented with stage I, II, III and IV, respectively. Additionally, 164 (91.1\%) received concurrent chemotherapy, and 163 (90.6\%) received neoadjuvant or adjuvant chemotherapy. The matched cases in both groups had balanced characteristics (all $P>0.05$ ). Table 1 summarized the clinical characteristics of the two arms.

\section{Survival outcomes}

The median follow-up time was 70 months (range, 10166 months) for the entire population, 107 months (range, 10-166 months) for the CV-IMRT arm and 50 months (range, 11-78 months) for the RV-IMRT arm, respectively. At the time of the last follow-up, 17 (9.4\%), $21(11.7 \%)$ and 35 (19.4\%) cases had developed locoregional recurrence, distant metastasis and disease progress, respectively. Three patients had developed both distant metastasis and recurrence. Eleven (12.2\%) cases of locoregional failure occurred in the CV-IMRT group with ten failed only in nasopharynx and one failed both in nasopharynx and regional nodal. In the RV-IMRT arm, five cases failed only in nasopharynx, and one failed both in nasopharynx and regional nodal. Of the total 17 locoregional failures, most cases $(76.5 \%)$ were in-field failures. With regard to the 21 distant metastasis cases, six patients had multiorgan metastasis, and 15 developed metastasis in an organ: 7 cases in lung, 4 cases in bone. Table 2 summarized the failure patterns.

As shown in Fig. 1, none statistically significant survival difference was observed in pairwise comparison between groups. For the CV-IMRT arm, the 3-year OS, PFS, DMFS, LRFS, RRFS and LRRFS rates were 88.9, 84.4, 92.2, 91.1, 98.9 and 91.1\%, respectively. For the RVIMRT arm, the 3-year OS, PFS, DMFS, LRFS, RRFS and LRRFS rates were 92.2, 85.6, 90.0, 93.3, 98.9 and 93.3\%, respectively. According to the subgroup analyses (Table 3), there was no significant survival difference between the CV-IMRT and RV-IMRT arms, irrespective of sex, age, $\mathrm{T}$ stage, $\mathrm{N}$ stage and clinical stage.

In multivariate Cox regression analyses, the predict value of various potential prognostic factors including gender, age, $\mathrm{T}$ stage, $\mathrm{N}$ stage, clinical stage, use of 
Table 1 Patient clinical characteristics

\begin{tabular}{|c|c|c|c|}
\hline \multirow[t]{2}{*}{ Characteristic } & CV-IMRT & RV-IMRT & \multirow[t]{2}{*}{$P$ value } \\
\hline & $N=90$ & $N=90$ & \\
\hline Gender & & & 0.864 \\
\hline Male & 66 & 68 & \\
\hline Female & 24 & 22 & \\
\hline Age & & & 1 \\
\hline$<45$ & 40 & 40 & \\
\hline$\geq 45$ & 50 & 50 & \\
\hline T stage & & & 0.976 \\
\hline $\mathrm{T} 1$ & 20 & 23 & \\
\hline $\mathrm{T} 2$ & 32 & 27 & \\
\hline T3 & 17 & 18 & \\
\hline T4 & 21 & 22 & \\
\hline N stage & & & 0.889 \\
\hline No & 13 & 12 & \\
\hline N1 & 25 & 26 & \\
\hline N2 & 45 & 44 & \\
\hline N3 & 7 & 8 & \\
\hline Clinical stage & & & 0.917 \\
\hline । & 6 & 6 & \\
\hline$\|$ & 16 & 16 & \\
\hline III & 42 & 41 & \\
\hline IV & 26 & 27 & \\
\hline Concurrent chemotherapy & & & 0.794 \\
\hline No & 9 & 7 & \\
\hline Yes & 81 & 83 & \\
\hline $\begin{array}{l}\text { Neoadjuvant/adjuvant } \\
\text { chemotherapy }\end{array}$ & & & 1 \\
\hline No & 9 & 8 & \\
\hline Yes & 81 & 82 & \\
\hline
\end{tabular}

CV-IMRT conventional-volume intensity-modulated radiation therapy, RV-IMRT reduced-volume intensity-modulated radiation therapy

Table 2 Failure patterns in the 180 patients after treatment

\begin{tabular}{lll}
\hline Patterns of failure & CV-IMRT n (\%) & RV-IMRT n (\%) \\
\hline Recurrence & $11(12.2 \%)$ & $6(6.7 \%)$ \\
NP recurrence only & $10(11.1 \%)$ & $5(5.6 \%)$ \\
LN regions recurrence only & 0 & 0 \\
NP and LN regions recurrence & $1(1.1 \%)$ & $1(1.1 \%)$ \\
In-field failures & $8(8.9 \%)$ & $5(5.6 \%)$ \\
Marginal failures & $1(1.1 \%)$ & $1(1.1 \%)$ \\
Out-field failures & $2(2.2 \%)$ & 0 \\
Distant metastasis & $10(11.1 \%)$ & $11(12.2 \%)$ \\
Distant metastasis and recurrence & $1(1.1 \%)$ & $2(2.2 \%)$ \\
\hline
\end{tabular}

CV-IMRT conventional-volume intensity-modulated radiation therapy, RV-IMRT reduced-volume intensity-modulated radiation therapy, $N P$ nasopharynx, $L N$ lymph node chemotherapy and radiotherapy pattern were evaluated (Table 4). Higher age was associated with high risk ratio for $\mathrm{OS}(\mathrm{RR}=2.408,95 \% \mathrm{CI}, 1.037-5.595 ; P=0.041)$. Beyond that, no prognostic factor was significant for survival.

\section{Toxicity}

All 180 patients tolerated well and completed the planned treatment therapy. Table 5 showed the radiation toxicity profiles for both groups. With regard to late toxicities, nine patients had Grade 3/4 skin reaction and 13 patients suffered from Grade 3/4 hearing loss. Specially, two patients of the CV-IMRT group and one of the RV-IMRT group bore temporal lobe injury. One of the RV-IMRT developed second primary tumor. Overall, the RV-IMRT was associated with significantly reduced risk of late xerostomia $(P=0.039)$ and hearing loss $(P=0.008)$, compared with the CV-IMRT.

In the subsequent subgroup analyses (Table 6), we tried to explore the potential population who might benefit from RV-IMRT with lower toxicity incidence. In terms of the radiation toxicity, the advantage of RVIMRT was mainly observed in patients with T1-2 stage, N0-1 stage and I-II stage. For T1-2 stage individuals, significant lower rates of late xerostomia $(P=0.008)$ and hearing loss $(P=0.01)$ were noted in the RV-IMRT group than those in the CV-IMRT group.

\section{Discussion}

Based on our study findings, generally speaking, the use of RV-IMRT for the treatment of nasopharyngeal carcinoma achieved similar treatment outcomes and did reduce the incidence of toxicity reactions compared versus CV-IMRT.

Since the early 1970s, traditional radiotherapy has been used for the treatment of NPC. The radiation technology is simple and imprecise, and radiation field encompassed in a two-dimensional portal is often large. Inevitably, various radiation-induced toxicities negatively affect patients' quality of life. With the rapid development, IMRT has been effectively utilized in the treatment of NPC and has been regarded as a standard modality. This technique satisfies the possibility of improving survival rates and protecting the adjacent normal structures simultaneously [4-6]. Since IMRT and chemotherapy has obviously enhanced survival rates and lengthened survival time [7-10, 20-23], professors turn to the decrease of treatment toxicity and the improvement of patients' quality of life. Yet, the delineation of clinical target volume in IMRT for nasopharyngeal carcinoma was largely derived from our experience of traditional radiotherapy, which is apparently improper in the precise radiation technology context. The dose coverage of the peripheral regions was suboptimal in the 

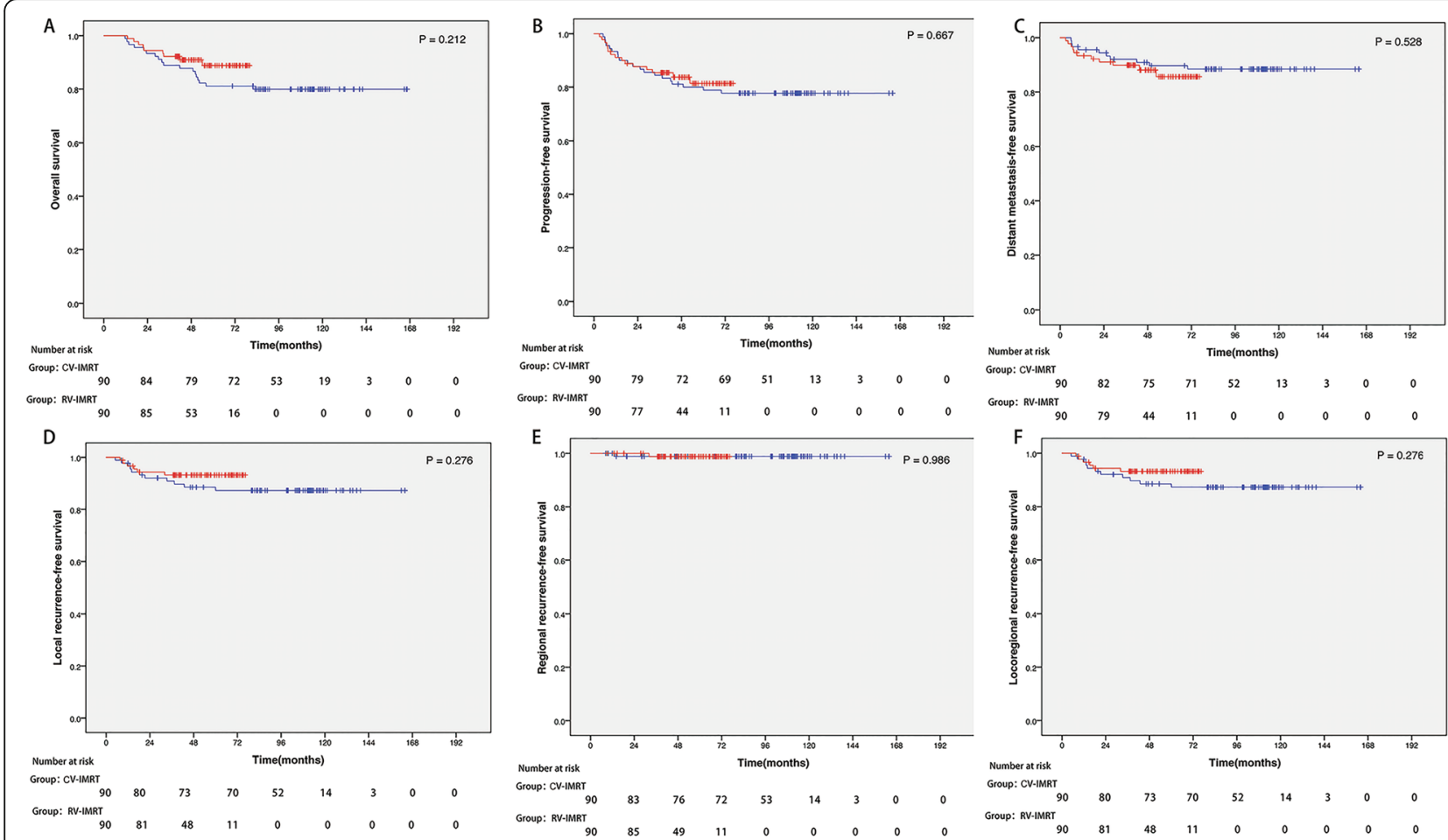

Fig. 1 Kaplan-Meier curves illustrate the survival of patients treated with IMRT. Conventional-volume intensity-modulated radiation therapy (blue line) and reduced-volume intensity-modulated radiation therapy (red line), including (a) overall survival, (b) progression-free survival, (c) distant metastasis-free survival, (d) local recurrence-free survival, (e) regional recurrence-free survival and (f) locoregional recurrence-free survival

traditional radiotherapy era, however, isolated recurrence in those areas was rare [13-15]. In other words, the treatment volume that adjacent to the primary disease was excessive. Additionally, a variety of toxicities came along with. The reduction of the clinical target volume should be taken into account.

A study by Lin et al. [16] has reported 323 cases of non-metastatic NPC receiving IMRT using reduced clinical target volume. The definition of CTV in this reduced-volume IMRT protocol was substantially reduced when comparing with that in the RTOG protocol, as described in our study. With a median follow-up of 30 months, the 3-year LRFS, RRFS, DMFS, DFS and OS rates were $95,98,90,85$, and $90 \%$, respectively. Besides, no Grade 3 or 4 xerostomia was detected beyond 3 months after the completion of treatment. In 2014, an

Table 3 Effects of subgroups on survival rates in nasopharyngeal carcinoma underwent CV-IMRT versus RV-IMRT

\begin{tabular}{|c|c|c|c|c|c|c|c|c|c|c|c|c|c|}
\hline \multirow[t]{2}{*}{ Variate } & & \multicolumn{3}{|c|}{ 3-year OS } & \multicolumn{3}{|c|}{ 3-year PFS } & \multicolumn{3}{|c|}{ 3-year DMFS } & \multicolumn{3}{|c|}{ 3-year LRRFS } \\
\hline & & CV-IMRT & RV-IMRT & $P$ & CV-IMRT & RV-IMRT & $P$ & CV-IMRT & RV-IMRT & $P$ & CV-IMRT & RV-IMRT & $P$ \\
\hline \multirow[t]{2}{*}{ Sex } & Female & $91.7 \%$ & $95.5 \%$ & 1 & $91.7 \%$ & $95.5 \%$ & 1 & $100.0 \%$ & $95.5 \%$ & 0.478 & $91.7 \%$ & $100.0 \%$ & 0.49 \\
\hline & Male & $87.9 \%$ & $91.2 \%$ & 0.583 & $81.8 \%$ & $82.4 \%$ & 1 & $89.4 \%$ & $88.2 \%$ & 1 & $90.9 \%$ & $91.2 \%$ & 1 \\
\hline \multirow[t]{2}{*}{ Age } & $<45$ & $95.0 \%$ & $90.0 \%$ & 0.675 & $95.0 \%$ & $80.0 \%$ & 0.087 & $97.5 \%$ & $85.0 \%$ & 0.108 & $97.5 \%$ & $90.0 \%$ & 0.359 \\
\hline & $\geq 45$ & $84.0 \%$ & $94.0 \%$ & 0.2 & $76.0 \%$ & $90.0 \%$ & 0.108 & $88.0 \%$ & $94.0 \%$ & 0.487 & $86.0 \%$ & $96.0 \%$ & 0.16 \\
\hline \multirow[t]{2}{*}{ T stage } & $\mathrm{T} 1-2$ & $86.5 \%$ & $94.0 \%$ & 0.319 & $82.7 \%$ & $88.0 \%$ & 0.579 & $90.4 \%$ & $94.0 \%$ & 0.716 & $92.3 \%$ & $94.0 \%$ & 1 \\
\hline & T3-4 & $92.1 \%$ & $90.0 \%$ & 1 & $86.8 \%$ & $82.5 \%$ & 0.756 & $94.7 \%$ & $85.0 \%$ & 0.264 & $89.5 \%$ & $92.5 \%$ & 0.708 \\
\hline \multirow[t]{2}{*}{$\mathrm{N}$ stage } & No-1 & $97.4 \%$ & $100.0 \%$ & 1 & $94.7 \%$ & $92.1 \%$ & 1 & $97.4 \%$ & $94.7 \%$ & 1 & $97.4 \%$ & $94.7 \%$ & 1 \\
\hline & N2-3 & $82.7 \%$ & $86.5 \%$ & 0.787 & $76.9 \%$ & $80.8 \%$ & 0.811 & $88.5 \%$ & $86.5 \%$ & 1 & $86.5 \%$ & $92.3 \%$ & 0.526 \\
\hline \multirow[t]{2}{*}{ Clinical stage } & $|-| \mid$ & $100.0 \%$ & $100.0 \%$ & - & $95.5 \%$ & $95.5 \%$ & 1 & $95.5 \%$ & $100.0 \%$ & 1 & $100.0 \%$ & $95.5 \%$ & 1 \\
\hline & III-IV & $85.3 \%$ & $89.7 \%$ & 0.605 & $80.9 \%$ & $82.4 \%$ & 1 & $91.2 \%$ & $86.8 \%$ & 0.585 & $88.2 \%$ & $92.6 \%$ & 0.561 \\
\hline
\end{tabular}


Table 4 Multivariate analysis of prognostic factors

\begin{tabular}{|c|c|c|c|c|c|c|c|c|}
\hline \multirow[t]{2}{*}{ Variate } & \multicolumn{2}{|l|}{ OS } & \multicolumn{2}{|l|}{ PFS } & \multicolumn{2}{|l|}{ DMFS } & \multicolumn{2}{|l|}{ LRRFS } \\
\hline & $\mathrm{RR}(95 \% \mathrm{Cl})$ & $P$ & $\mathrm{RR}(95 \% \mathrm{Cl})$ & $P$ & $\mathrm{RR}(95 \% \mathrm{Cl})$ & $P$ & $\mathrm{RR}(95 \% \mathrm{Cl})$ & $P$ \\
\hline \multicolumn{9}{|l|}{ Group } \\
\hline RV-IMRT vs. CV-IMRT & $0.580(0.257-1.309)$ & 0.19 & $0.826(0.420-1.622)$ & 0.578 & $1.285(0.538-3.070)$ & 0.573 & $0.553(0.203-1.502)$ & 0.245 \\
\hline \multicolumn{9}{|l|}{ Gender } \\
\hline Male vs. Female & $0.970(0.382-2.464)$ & 0.95 & $1.918(0.736-4.998)$ & 0.183 & $2.979(0.684-12.978)$ & 0.146 & $1.563(0.439-5.562)$ & 0.49 \\
\hline \multicolumn{9}{|l|}{ Age } \\
\hline$\geq 45$ vs. $<45$ & $2.408(1.037-5.595)$ & 0.041 & $1.451(0.724-2.908)$ & 0.294 & $1.053(0.438-2.528)$ & 0.909 & $1.607(0.575-4.490)$ & 0.365 \\
\hline \multicolumn{9}{|l|}{ T stage } \\
\hline T3-4 vs. T1-2 & $0.817(0.340-1.964)$ & 0.651 & $0.916(0.415-2.021)$ & 0.828 & $1.068(0.396-2.885)$ & 0.896 & $1.179(0.368-3.776)$ & 0.781 \\
\hline \multicolumn{9}{|l|}{ N stage } \\
\hline N2-3 vs. N0-1 & $2.455(0.649-9.279)$ & 0.186 & $2.192(0.687-6.992)$ & 0.185 & $2.715(0.556-13.257)$ & 0.217 & $1.490(0.361-6.159)$ & 0.581 \\
\hline \multicolumn{9}{|l|}{ Clinical stage } \\
\hline III-IV vs. I-II & $3.280(0.281-38.226)$ & 0.343 & $0.926(0.183-4.683)$ & 0.926 & $0.730(0.080-6.639)$ & 0.78 & $1.165(0.129-10.480)$ & 0.892 \\
\hline \multicolumn{9}{|c|}{ Neoadjuvant/adjuvant chemotherapy } \\
\hline Yes vs. No & $0.600(0.085-4.238)$ & 0.608 & $0.695(0.108-4.464)$ & 0.702 & $1.764(0.084-36.960)$ & 0.715 & $0.404(0.047-3.457)$ & 0.408 \\
\hline \multicolumn{9}{|c|}{ Concurrent chemotherapy } \\
\hline Yes vs. No & $0.987(0.041-23.701)$ & 0.994 & $3.338(0.237-46.915)$ & 0.371 & $0.796(0.030-20.935)$ & 0.891 & - & - \\
\hline
\end{tabular}

OS overall survival, PFS progression-free survival, DMFS distant metastasis-free survival, LRRFS locoregional recurrence-free survival, CV-IMRT conventional-volume intensity-modulated radiation therapy, RV-IMRT reduced-volume intensity-modulated radiation therapy

update [24] of the reduced-volume IMRT analyzed 414 NPC patients: the 5-year LRFS, RRFS, DMFS, DFS and OS rates were $95,97,82,77$, and $80 \%$, respectively. The survival results of these studies were comparative with that of other researches [25-27] used a relative largevolume IMRT by taking RTOG protocol as reference. This implied that using RV-IMRT in the treatment of NPC was safe and effective. Nevertheless, there has not been direct comparison between the two protocols.

In this study, we performed a retrospective analysis to compare the clinical treatment outcomes and toxicities of RV-IMRT with those of CV-IMRT for NPC patients. As for failure patterns, distant metastasis was the main failure mode with a rate of $11.7 \%$ in the whole population, locoregional recurrence accounted for $9.4 \%$ with the majority were in-field failures. None significant survival difference was shown between the two groups, irrespective of sex, age, $\mathrm{T}$ stage, $\mathrm{N}$ stage and clinical stage. Additionally, NPC patients who received RVIMRT in our study had similar survival rates with those in the study by Lin et al. [16] (3-year OS, 92.2\% vs. 90\%, 3-year DMFS, $90.0 \%$ vs. 90\%; 3-year LRFS, 93.3\% vs. 95\%; 3-year RRFS, $98.9 \%$ vs. 98\%, respectively).

According to the univariate analyses, there were significant survival differences (OS, $P=0.007$; PFS, $P=0.02$ ) between different $\mathrm{N}$ category (N0-1 vs. N2-3). However, the multivariate analyses showed that neither $\mathrm{T}$ stage nor $\mathrm{N}$ stage was significant to predict survival outcomes. In addition, a number of papers [28-31] have also indicated that there was no significant survival difference between each T stage in NPC patients treated with IMRT. However, it should be noted that tumors were staged according to the clinical staging system based on data from conventional 2D-RT in these papers. We suspected that

Table 5 Radiation toxicity profiles

\begin{tabular}{|c|c|c|c|c|c|c|c|c|c|c|c|}
\hline \multirow[t]{2}{*}{ Late toxicity } & \multicolumn{5}{|c|}{ CV-IMRT $(n=90)$} & \multicolumn{5}{|c|}{$\operatorname{RV}-\operatorname{IMRT}(n=90)$} & \multirow[t]{2}{*}{$P$} \\
\hline & Grade 0 & Grade 1 & Grade 2 & Grade 3 & Grade 4 & Grade 0 & Grade 1 & Grade 2 & Grade 3 & Grade 4 & \\
\hline Xerostomia & 12 & 60 & 18 & 0 & 0 & 20 & 60 & 10 & 0 & 0 & 0.039 \\
\hline Mucositis & 16 & 64 & 10 & 0 & 0 & 20 & 62 & 8 & 0 & 0 & 0.406 \\
\hline Skin reaction & 7 & 54 & 24 & 5 & 0 & 5 & 60 & 21 & 4 & 0 & 0.714 \\
\hline Dysphagia & 12 & 70 & 8 & 0 & 0 & 15 & 69 & 6 & 0 & 0 & 0.432 \\
\hline Hearing loss & 21 & 47 & 14 & 7 & 1 & 34 & 46 & 5 & 5 & 0 & 0.008 \\
\hline Blurred vision & 81 & 8 & 1 & 0 & 0 & 84 & 4 & 2 & 0 & 0 & 0.441 \\
\hline
\end{tabular}


Table 6 Effects of subgroups on late toxicity in nasopharyngeal carcinoma underwent CV-IMRT versus RV-IMRT

\begin{tabular}{|c|c|c|c|c|c|c|c|}
\hline \multirow[t]{2}{*}{ Variate } & & \multicolumn{6}{|l|}{$P$ value } \\
\hline & & Xerostomia & Mucositis & Skin reaction & Dysphagia & Hearing loss & Blurred vision \\
\hline \multirow[t]{2}{*}{ Sex } & Female & 0.511 & 0.562 & 0.362 & 0.36 & 0.077 & 0.625 \\
\hline & Male & 0.038 & 0.145 & 0.893 & 0.716 & 0.042 & 0.532 \\
\hline \multirow[t]{2}{*}{ Age } & $<45$ & 0.483 & 0.831 & 0.613 & 0.32 & 0.023 & 0.327 \\
\hline & $\geq 45$ & 0.036 & 0.384 & 0.215 & 0.838 & 0.12 & 0.756 \\
\hline \multirow[t]{2}{*}{ T stage } & $\mathrm{T} 1-2$ & 0.008 & 0.348 & 0.987 & 0.97 & 0.01 & 0.06 \\
\hline & T3-4 & 0.995 & 0.717 & 0.542 & 0.252 & 0.253 & 0.272 \\
\hline \multirow[t]{2}{*}{$\mathrm{N}$ stage } & NO-1 & 0.255 & 0.546 & 0.676 & 0.787 & 0.01 & 0.969 \\
\hline & N2-3 & 0.079 & 0.553 & 0.81 & 0.236 & 0.185 & 0.252 \\
\hline \multirow[t]{2}{*}{ Clinical stage } & $|-| \mid$ & 0.269 & 0.373 & 0.78 & 1 & 0.011 & 0.607 \\
\hline & III-IV & 0.087 & 0.635 & 0.765 & 0.353 & 0.099 & 0.779 \\
\hline \multirow[t]{2}{*}{ Neoadjuvant/adjuvant chemotherapy } & No & 0.715 & 0.897 & 0.363 & 0.675 & 0.017 & 1 \\
\hline & Yes & 0.036 & 0.406 & 0.86 & 0.318 & 0.049 & 0.585 \\
\hline \multirow[t]{2}{*}{ Concurrent chemotherapy } & No & 0.419 & 0.605 & 0.402 & 0.696 & 0.003 & - \\
\hline & Yes & 0.053 & 0.492 & 0.831 & 0.318 & 0.069 & 0.412 \\
\hline
\end{tabular}

CV-IMRT conventional-volume intensity-modulated radiation therapy, RV-IMRT reduced-volume intensity-modulated radiation therapy

prognostic factors may vary with the progression of diagnostic and treatment techniques. Advanced imaging techniques can early detect occult metastases and accurately define the extent of tumor invasion. The application of IMRT and chemotherapy in the treatment of NPC has improved survival conditions obviously. As a consequence, the accuracy and applicability of staging systems should be reevaluated with the rapid development of imaging techniques and therapeutic methods. The latest eighth edition of the UICC/AJCC cancer staging classification was based on data in the IMRT era, which may perform better in predicting survival outcomes. Moreover, the multivariate analyses showed that receiving chemotherapy (concurrent chemotherapy, neoadjuvant or adjuvant chemotherapy) has no predictive value for treatment outcomes. Seemingly, it was not in line with our experience. We noticed that only a tiny proportion of patients included in this study have not received chemotherapy and the majority of which were presented with stage I. Early stage NPC cases treated with radiotherapy alone can be rendered disease-free in the long term. These could lead to the negative result.

Although a plenty of studies have substantiated that various radiation-induced toxicities are obviously reduced by using IMRT, the incidence of SPT is inverse. IMRT is likely to double the incidence of SPT compared versus $2 \mathrm{D}-\mathrm{RT}$ from 1 to $1.75 \%$ for NPC patients [32, 33]. At the time of the last follow-up, however, only one patient $(0.56 \%)$ developed SPT in our study. Overall, the RV-IMRT was associated with significantly reduced risk of late xerostomia $(P=0.039)$ and hearing loss $(P=0.008)$, compared versus the CV-IMRT. In the subgroup analyses, we tried to explore the potential population who might benefit from RV-IMRT. Generally, patients with T1-2 stage, N0-1 stage and I-II stage disease particularly benefit from RV-IMRT with similar survival rates and lower toxicity incidence. Hence, dosimetric improvements indeed translate into improvements in adverse events.

Despite of these satisfactory outcomes, the selection and definition of CTV in IMRT for NPC is far from addressed. Our study is supposed to has a certain guiding significance to the delineation of target volumes. Meanwhile, there are several limitations in our study. Firstly, the study was arranged as a retrospective trial with a small amount of patients. In spite of the well distributed patients, this study was performed in a nonendemic setting with relatively small amounts of cases. Furthermore, the small number of patients included may lead to an inadequate number of events needed for further analysis and limit the accuracy of the research results. Given all these, the generalization of the conclusions needs to be carefully considered, and well-designed trials are needed to confirm the findings in the future. Secondly, the median follow-up time was 50 months (range, 11-78 months) for the RV-IMRT arm. Since the majority of recurrence occurs in the first 2 years after the completion of radiotherapy [34-36], a median follow-up of 50 months signified that the true incidence of recurrence in the RV-IMRT arm may approximate our findings. With regard to the long term overall survival rate, longer follow-ups are in great need to evaluate the efficacy of RV-IMRT in the treatment of NPC. Thirdly, patients were paired using propensity score matching method to 
reduce imbalance between the experimental and control groups, thereby reducing the potential for bias. It should be noted that this method is of a few limitations. The weakness of PSM is that it does not consider the interaction between variables, but only focuses on the effect of a certain variable. Besides, after pruning some observations, the remaining samples may not be representative. Finally, the enrollment interval was longer than 10 years, many crucial factors such as the quality of image have varied during this period. The survival outcomes may be influenced by these factors.

\section{Conclusions}

This study indicated that the use of RV-IMRT for the treatment of nasopharyngeal carcinoma did not adversely impact survival rates but did reduce the incidence of radiation toxicity compared versus the CVIMRT. The delineation of target volumes in IMRT for nasopharyngeal carcinoma still needs to be optimized.

\begin{abstract}
Abbreviations
2D-RT: Two-dimensional radiotherapy; AJCC: The American Joint Committee on Cancer; CT: Computed tomography; CTCAE: The Common Terminology Criteria for Adverse Events; CTV: Clinical target volume; CV-

IMRT: Conventional-volume IMRT; DMFS: Distant metastasis-free survival; GTV: Gross tumor volume; IMRT: Intensity modulated radiation therapy; LN: Lymph node; LRFS: Local recurrence-free survival; LRRFS: Locoregional recurrence-free survival; MRI: Magnetic resonance imaging; NP: Nasopharynx; NPC: Nasopharyngeal carcinoma; OAR: Organ at risk; OS: Overall survival; PFS: Progression-free survival; PSM: Propensity score matching; PTV: Planning target volume; RRFS: Regional recurrence-free survival; RTOG: The Radiation Therapy Oncology Group; RV-IMRT: Reduced-volume IMRT; SPT: Second primary tumor
\end{abstract}

\section{Acknowledgements}

Not applicable.

\begin{abstract}
Authors' contributions
$L L, L T$, and $L F$ conceived the study, participated in the study design. LF, JT, and $Z J$ analyzed and interpreted the data. XZ and YR helped to data acquisition and statistical analysis. WX and XL interpreted the results. LF and $\mathrm{ZH}$ drafted the initial manuscript. LP and WF revised the manuscript. All authors read and approved the final manuscript.
\end{abstract}

\section{Funding}

Not applicable.

\section{Availability of data and materials}

The datasets used and analysed during the current study are available from the corresponding author on reasonable request.

\section{Ethics approval and consent to participate}

This study was carried out in accordance with the Declaration of Helsinki. In view of the retrospective nature of our study, it was difficult to obtain consent from all patients. None recognizable patient information was used in this study. And we acquired an exemption from ethics approval conferred by the Subcommittee on Biomedical Ethics of West China Hospital.

\section{Consent for publication}

Not applicable.

\section{Competing interests}

The authors declare that they have no competing interests.

\section{Author details}

'Department of Radiation Oncology, Cancer Center, State Key Laboratory of Biotherapy, West China Hospital, Sichuan University, Chengdu 610041, Sichuan, China. ${ }^{2}$ Mammary Oncology and Department of Medical Oncology, Clinical Research Center for Breast, Cancer Center, Laboratory of Molecular Diagnosis of Cancer, West China Hospital, Sichuan University, Chengdu 610041, Sichuan, China. ${ }^{3}$ Cancer Center, State Key Laboratory of Biotherapy, West China Hospital, Sichuan University, Chengdu 610041, Sichuan, China.

Received: 14 November 2018 Accepted: 29 May 2019

Published online: 08 June 2019

\section{References}

1. Cao S-M, Simons MJ, Qian C-N. The prevalence and prevention of nasopharyngeal carcinoma in China. Chin J Cancer. 2011;30(2):114-9.

2. Trejaut J, Lee CL, Yen JC, Loo JH, Lin M. Ancient migration routes of Austronesian-speaking populations in oceanic Southeast Asia and Melanesia might mimic the spread of nasopharyngeal carcinoma. Chin J Cancer. 2011;30(2):96.

3. NCCN Clinical Practice Guidelines in Oncology (NCCN Guidelines) [https:// www.nccn.org/professionals/physician_gls/pdf/head-and-neck.pdf].

4. Toledano I, Graff P, Serre A, Boisselier P, Bensadoun RJ, Ortholan C, Pommier P, Racadot S, Calais G, Alfonsi M. Intensity-modulated radiotherapy in head and neck cancer: results of the prospective study GORTEC 2004-03. Radiother Oncol. 2012;103(1):57-62.

5. Su SF, Han F, Zhao C, Chen CY, Xiao WW, Li JX, Lu TX. Long-term outcomes of early-stage nasopharyngeal carcinoma patients treated with intensitymodulated radiotherapy alone. Int J Radiat Oncol Biol Phys. 2012;82(1):327-33.

6. Fu-Min F, Chih-Yen C, Wen-Ling T, Hui-Chun C, Hsuan-Chih H, Chun-Chung L, Tai-Lin H, Hsuan-Ying H. Quality of life and survival outcome for patients with nasopharyngeal carcinoma receiving three-dimensional conformal radiotherapy vs. intensity-modulated radiotherapy-a longitudinal study. Int J Radiat Oncol Biol Phys. 2008;72(2):356-64.

7. Wong FCS, Ng AWY, Lee VHF, Lui CMM, Yuen KK, Sze WK, Leung TW, Tung SY. Whole-field simultaneous integrated-boost intensity-modulated radiotherapy for patients with nasopharyngeal carcinoma. Int J Radiat Oncol Biol Phys. 2010;76(1):138-45.

8. Wolden SL, Chen WC, Pfister DG, Kraus DH, Berry SL, Zelefsky MJ. Intensitymodulated radiation therapy (IMRT) for nasopharynx cancer: update of the memorial Sloan-Kettering experience. Int J Radiat Oncol Biol Phys. 2006; 64(1):57-62

9. Wai Tong N, Lee MCH, Wai Man H, Cheuk Wai C, Kin Chung L, Chan OSH, Lee AWM. Clinical outcomes and patterns of failure after intensitymodulated radiotherapy for nasopharyngeal carcinoma. Int J Radiat Oncol Biol Phys. 2011;79(2):420-8.

10. Peng G, Wang T, Yang KY, Zhang S, Zhang T, Li Q, Han J, Wu G. A prospective, randomized study comparing outcomes and toxicities of intensity-modulated radiotherapy vs. conventional two-dimensional radiotherapy for the treatment of nasopharyngeal carcinoma. Radiother Oncol. 2012;104(3):286-93.

11. Nancy $L$, Jonathan $H$, Garden AS, William S, Bonnie G, Ping X, Walter B, Morrison WH, Jeanne $Q$, Wade T. Intensity-modulated radiation therapy with or without chemotherapy for nasopharyngeal carcinoma: radiation therapy oncology group phase II trial 0225. J Clin Oncol. 2009;27(22):3684.

12. Lee NY, Zhang Q, Pfister DG, Kim J, Garden AS, Mechalakos J, Hu K, Le QT, Colevas AD, Glisson BS. Addition of bevacizumab to standard chemoradiation for locoregionally advanced nasopharyngeal carcinoma (RTOG 0615): a phase 2 multi-institutional trial. Lancet Oncol. 2012;13(2):172-80.

13. Chau RM, Teo PM, Choi PH, Cheung KY, Lee WY. Three-dimensional dosimetric evaluation of a conventional radiotherapy technique for treatment of nasopharyngeal carcinoma. Radiother Oncol. 2001;58(2):143-53.

14. Chau RMC, Teo PML, Kam MKM, Leung SF, Cheung KY, Chan ATC. Dosimetric comparison between 2-dimensional radiation therapy and intensity modulated radiation therapy in treatment of advanced T-stage nasopharyngeal carcinoma: to treat less or more in the planning organ-at-risk volume of the brainstem and spinal cord. Med Dosim. 2008;32(4):263-70.

15. Dawson LA, Anzai Y, Marsh L, Martel MK, Paulino A, Ship JA, Eisbruch A. Patterns of local-regional recurrence following parotid-sparing conformal and segmental intensity-modulated radiotherapy for head and neck cancer. Int J Radiat Oncol Biol Phys. 2000;46(5):1117-26. 
16. Lin S, Pan J, Han L, Zhang X, Liao X, Lu JJ. Nasopharyngeal carcinoma treated with reduced-volume intensity-modulated radiation therapy: report on the 3-year outcome of a prospective series. Int J Radiat Oncol Biol Phys. 2009;75(4):1071-8.

17. Common Terminology Criteria for Adverse Events v4.0. [https://evs.nci.nih. gov/ftp1/CTCAE/CTCAE_4.03/CTCAE_4.03_2010-06-14_QuickReference_5x7. pdf]

18. D'Agostino RB. Propensity score methods for Bias reduction in the comparison of a treatment to a non-randomized control group. Stat Med. 1998;17(19):2265-81.

19. Kaplan EL, Meier P. Nonparametric estimation from incomplete observations. J Am Stat Assoc. 1958;53(282):457-81.

20. Al-Sarraf M, Leblanc M, Giri PG, Fu KK, Cooper J, Vuong T, Forastiere AA, Adams G, Sakr WA, Schuller DE. Chemoradiotherapy versus radiotherapy in patients with advanced nasopharyngeal cancer: phase III randomized intergroup study 0099. J Clin Oncol. 1998;16(4):1310-7.

21. Chan ATC, Leung SF, Ngan RKC, Teo PML, Lau WH, Kwan WH, Hui EP, Yiu HY, Winnie Y, Cheung FY. Overall survival after concurrent cisplatinradiotherapy compared with radiotherapy alone in locoregionally advanced nasopharyngeal carcinoma. J Natl Cancer Inst. 2005;97(7):536-9.

22. Wu X, Huang PY, Peng PJ, Lu LX, Han F, Wu SX, Hou X, Zhao HY, Huang Y, Fang WF. Long-term follow-up of a phase III study comparing radiotherapy with or without weekly oxaliplatin for locoregionally advanced nasopharyngeal carcinoma. Ann Oncol. 2013;24(8):2131-6.

23. Binglan Z, Zeming $M$, Wei $D$, Yan $W$, Lei L, Yuquan $W$. Intensity-modulated radiation therapy versus $2 \mathrm{D}-\mathrm{RT}$ or $3 \mathrm{D}-\mathrm{CRT}$ for the treatment of nasopharyngeal carcinoma: a systematic review and meta-analysis. Oral Oncol. 2015;51(11):1041-6.

24. Lin S, Pan J, Han L, Guo Q, Hu C, Zong J, Zhang X, Lu JJ. Update report of nasopharyngeal carcinoma treated with reduced-volume intensitymodulated radiation therapy and hypothesis of the optimal margin. Radiother Oncol. 2014;110(3):385-9.

25. Sultanem K, Shu HK, Ping X, Akazawa C, Quivey JM, Verhey L, Fu KK. Threedimensional intensity-modulated radiotherapy in the treatment of nasopharyngeal carcinoma: the University of California-san Francisco experience. Int J Radiat Oncol Biol Phys. 2000;48(3):711-22.

26. Tham WK, Hee SW, Yeo MC, Salleh PB, Lee J, Tan WK, Fong KW, Chua ET, Wee TS. Treatment of nasopharyngeal carcinoma using intensity-modulated radiotherapy — the National Cancer Centre Singapore Experience. Int J Radiat Oncol Biol Phys. 2009;75(5):1481-6.

27. Kam MKM, Teo PML, Chau RMC, Cheung KY, Choi PHK, Kwan WH, Leung SF, Benny Z, Chan ATC. Treatment of nasopharyngeal carcinoma with intensitymodulated radiotherapy: the Hong Kong experience. Int J Radiat Oncol Biol Phys. 2004;60(5):1440-50

28. Lee N, Xia P, Akazawa P, Akazawa C, Quivey JM, Weinberg V, Fu KK. Intensity-modulated radiotherapy in the treatment of nasopharyngeal carcinoma: an update of the UCSF experience. Int J Radiat Oncol Biol Phys. 2002;53(1):12-22.

29. Lee AWM, Lau WH, Tung SY, Chua DTT, Rick C, Xu L, Lillian S, Sze WM, Leung TW, Sham JST. Preliminary results of a randomized study on therapeutic gain by concurrent chemotherapy for regionally-advanced nasopharyngeal carcinoma: NPC-9901 Trial by the Hong Kong Nasopharyngeal Cancer Study Group. J Clin Oncol. 2005;23(28):6966-75.

30. Yan-Ping M, Fang-Yun X, Li-Zhi L, Ying S, Li L, Ling-Long T, Xin-Biao L, Hong-Yao X, Lei C, Shu-Zhen L. Re-evaluation of 6th edition of AJCC staging system for nasopharyngeal carcinoma and proposed improvement based on magnetic resonance imaging. Int J Radiat Oncol Biol Phys. 2009; 73(5):1326-34

31. Lu H, Lin SJ, Pan JJ, Chen CB, Yu Z, Zhang XC, Liao XY. Prognostic factors of 305 nasopharyngeal carcinoma patients treated with intensity-modulated radiotherapy. Chin J Cancer. 2010;29(2):145-50.

32. Hall EJ, Wuu CS. Radiation-induced second cancers: the impact of 3D-CRT and IMRT. Int J Radiat Oncol Biol Phys. 2003;56(1):83-8.

33. Lin K, Jiade Jay L, Chaosu H, Xiaomao G, Yongru W, Youwang Z. The risk of second primary tumors in patients with nasopharyngeal carcinoma after definitive radiotherapy. Cancer. 2006;107(6):1287-93.

34. Sanguineti G, Geara FB, Garden AS, Tucker SL, Ang KK, Morrison WH, Peters $\sqcup$. Carcinoma of the nasopharynx treated by radiotherapy alone: determinants of local and regional control. Int J Radiat Oncol Biol Phys. 1997:37(5):985-96.
35. Vikram B, Mishra UB. Pattern of failure in carcinoma of the nasopharynx: I. failure at the primary site. Int J Radiat Oncol Biol Phys. 1985;11(8):1455-9.

36. Bedwinek JM, Perez CA, Keys DJ. Analysis of failures after definitive irradiation for epidermoid carcinoma of the nasopharynx. Cancer. 1980; 45(11):2725-9.

\section{Publisher's Note}

Springer Nature remains neutral with regard to jurisdictional claims in published maps and institutional affiliations.
Ready to submit your research? Choose BMC and benefit from:

- fast, convenient online submission

- thorough peer review by experienced researchers in your field

- rapid publication on acceptance

- support for research data, including large and complex data types

- gold Open Access which fosters wider collaboration and increased citations

- maximum visibility for your research: over $100 \mathrm{M}$ website views per year

At BMC, research is always in progress.

Learn more biomedcentral.com/submissions 